\title{
Transdisciplina y docencia universitaria
}

\author{
Araceli Martínez Arroyo \\ Universidad Iberoamericana Ciudad de México, México \\ araceli.martinez@ibero.mx \\ ORCID: https://orcid.org/0000-0002-3760-7792 \\ https://doi.org/10.48102/didac.2021..78_JUL-DIC.82
}

8

La publicación de este número 78 de la revista DIDAC ocurre en un contexto en el que los incipientes programas de vacunación alrededor del mundo y las acciones de reactivación económica atisban un ánimo esperanzador de respuesta frente a la pandemia ocasionada por la COVID-19 que desde hace más de un año nos ha sacudido. Evidentemente este ánimo de esperanza no se sostiene en una ingenuidad ante respuestas fáciles o salidas únicas, sino que, ante una clara - y dolorosa - conciencia de la brecha de desigualdad social, económica y educativa que se acentuado durante estos meses explora los caminos de contribución desde su propio lugar.

En este sentido, la conversación que se teje en torno a sus artículos advierte sobre la relevancia que adquieren las instituciones de educación superior $y$, en particular, las acciones de docentes como promotores de espacios formativos profesionales con sentido de respuesta social ante los problemas complejos que nos afectan.

Las experiencias docentes -ocurridas antes o durante el periodo de contingencia - así como los planteamientos de investigación que se exponen, provienen de trayectorias y ámbitos disciplinares y geográficos diversos. En ellos se expresan dificultades, retos y aprendizajes conceptuales y prácticos sobre la docencia transdisciplinar $y$, aún desde referentes y aproximaciones diferenciadas, se distinguen rasgos y planteamientos comunes en su conceptualización e implementación. Por ejemplo, sin negar la importancia del saber disciplinar, se enuncia su insuficiencia ante la urgencia de responder a problemas globales que requieren una mirada integral para atenderlos. Trascender la mirada disciplinar es un paso que, aunque parezca mínimo, representa un gran avance en la posibilidad de establer diálogos, acciones y consensos con otras disciplinas y, de manera fundamental, con otras personas y saberes más allá de las comunidades académicas y científicas. El concepto de la transdisciplina cuestiona la visión "academicocentrista" y los muros epistemológicos que la arropan, y plantea la necesidad de salir de éstos para generar y movilizar conocimientos con otros que permitan, no solamente la comprensión de un problema sino la búsqueda de su solución. La docencia universitaria, por tanto, no puede ser concebida como un asunto técnico de transmisión de conocimientos sino como una práctica transdisciplinar de cuidado común que abona a construir la justicia social.

En los artículos que integran este número, el lector se acercará o profundizará en su comprensión de un concepto en construcción como lo es el de la transdisciplina. Al mismo tiempo, reconocerá las múltiples posibilidades que ofrece la docencia universitaria en este camino para enriquecer sus prácticas y la pertinencia de su ser y actuar. Los diez artículos que participan de esta conversación -incluyen un texto en inglés con su traducción al español— ilustran múl- 
tiples ángulos de la transdisciplina en su relación con la docencia universitaria.

El artículo "El curso en Educación Basada en Evidencias (EBE): reflexiones para la transdisciplina, la docencia y la investigación" describe una experiencia de formación docente en la que participan profesores de distintas disciplinas y niveles educativos. El planteamiento sobre la docencia formulado en este texto la describe como una práctica disciplinar (en tanto, actúa desde conocimientos disciplinares del docente profesional) e interdisciplinar (en tanto que apela a conocimientos de índole didáctico-pedagógica adquiridos en la práctica de la docencia). Al mismo tiempo, enfatiza que la docencia es, indudablemente, una práctica transdisciplinar en la medida que posibilita la articulación de conocimientos de distinta naturaleza (incluidos los que no son académicos) para la comprensión y la solución creativa de grandes y complejos problemas. De acuerdo con los autores, en la articulación de estos conocimientos es que radica la propia definición de lo transdisciplinar y, por tanto, exige que los procesos de formación docente movilicen y trasladen conocimientos teóricos y técnicos a la práctica docente. En la experiencia descrita, los conocimientos teóricos y técnicos provienen de la investigación en educación y se trasladan a prácticas y contextos tan diversos como los de la propia población de docentes participantes. El proceso de formación docente expuesto en este artículo tomó como referencia los planteamientos de la investigación basada en evidencias así como las tres dimensiones del aprendizaje transdisciplinar planteadas por McGregor (2017, en Benavides et al., 2021): 1) Trascender y fusionar conocimientos de distinta naturaleza (disciplinares y no disciplinares); 2) Incluir procesos de actuación, observación e interpretación, en los que los distintos tipos de conocimientos involucrados no son superiores unos a otros, y 3) Conducir a hábitos mentales transdisciplinares que posibiliten un pensamiento integrador que reconoce las fronteras de los conocimientos y lo habilite a traspasarlas.

En "Una experiencia transdisciplinar de trabajo comunitario en contextos de formación profesio- nal", Pérez, Bravo y Mora advierten que ciertas características del actual modelo de formación universitaria, tales como la fragmentación de las áreas del conocimiento y la orientación prioritaria hacia el entrenamiento profesional, alejan a las instituciones de educación superior de su misión de transformación social. De acuerdo con los autores, sostener una mirada del mundo a través del lente disciplinar puede conducir a la generación y transmisión de un conocimiento parcial, lineal y lógico de la realidad, $y$, aunque no niegan la importancia de dicho conocimiento disciplinar, sí se advierte su insuficiencia cuando es indispensable mirar los fenómenos sociales como un entramado complejo, amplio y divergente. Dos planteamientos son fundamentales en la experiencia de formación profesional descrita. Por un lado, asumir un enfoque transdisciplinar del proceso de formación, entendido éste como el diálogo entre los distintos saberes —incluidos aquellos que surgen al interior de las propias comunidadesque conducen a prácticas de transformación local y regional. Por otro lado, articular dicho enfoque transdisciplinar a la experiencia de formación ciudadana en la que los jóvenes reconocen el rol que cumplen en los procesos de transformación social. Con la participación de estudiantes de distintas carreras organizados alrededor de un proyecto en comunidad que emplea la metodología de aprendizaje y servicio se movilizaron conocimientos y prácticas en experimentación y diálogo permanente entre todos los actores. Así, se buscó trascender y ampliar una visión reducida del ciudadano elector a la del ciudadano como actor social.

Las tensiones interdisciplinares generadas en la enseñanza de la historia de la ciencia para biólogos son expuestas en el artículo "Historia de la ciencia y su enseñanza: alcances (inter)disciplinares en la formación científica". Su autor, Rodríguez Caso, afirma que es el sentido interdisciplinar lo que debe orientar la formación de científicos a fin de enmarcar los conocimientos provenientes de las humanidades y las ciencias sociales en las facetas culturales de la práctica científica. El autor advierte los riesgos de enfoques historiográficos que pueden conducir a visiones 
sesgadas, tendenciosas y anacrónicas del conocimiento científico y reconoce que el conflicto en la en señanza interdisciplinar no se resuelve con la participación de diferentes especialistas en una clase o con la presencia de un profesor que "sepa de todo". A fin de evitar dogmatismos y planteamientos cientificistas o cientistas que alejan a la ciencia de la sociedad y la coloquen en lugar apto para "genios", el autor recurre a un enfoque social y cultural de la historia que obliga a interpretar a los autores y sus obras dentro de su contexto y en relación con otras discusiones y autores. Así, la historia deja de ser "una verdad" y se adopta una mirada más compleja ante ella. Más allá de discusiones teóricas o estrategias metodológicas, la actitud del docente —o del científico - en su acercamiento ante el conocimiento es fundamental. Rodríguez Caso propone apelar a la humildad —en términos epistémicos_ y orientar la práctica docente alrededor de ésta. Es, dicha humildad epistémica, la que permitiría reconocer que la construcción del conocimiento científico es contigente $y$, por tanto, deben evitarse posturas únicas. Es también a partir de este lugar que pueden generarse estrategias que reduzcan tensiones y posibiliten una formación para los científicos con mayor capacidad crítica y sentido social.

En el artículo de Juan Carlos Silas y Laura Angélica Bárcenas se ilustra que la práctica docente, cuando está orientada a la formación del compromiso social, es un lugar de acción más allá de límites técnicos o disciplinares. La investigación presentada en "Prácticas docentes que promueven el compromiso social de sus alumnos. Una visión interdisciplinar" expone los dos tipos de prácticas detectadas alrededor de las cuales se organiza dicha formación: por un lado, las discursivas y, por otro lado, las de acercamiento a la realidad. Las prácticas discursivas están integradas, a su vez, por seis prácticas docentes: 1) exposición y diálogo; 2) confrontación e ironía; 3) reflexión enfocada; 4) debate; 5) colaboración entre alumnos, y 6) lectura de textos). Las prácticas de acercamiento a docentes comprenden cuatro subtipos: 1) contacto directo; 2) aprendizaje situado; 3) trabajo localizado, y 4) vivencia o experiencia vicaria. La caracterización obtenida por los autores en voz de 49 docentes, pertenecientes a seis instituciones del Sistema Universitario Jesuita, permite reconocer el compromiso social como un contenido interdisciplinar que articula las interacciones entre docentes y alumnos y permite la toma de conciencia sobre otras realidades y las acciones posibles para mejorararlas.

El artículo en inglés de Wanner, Bernert, Fischer y Schmitt, así como su correspondiente traducción al español, describen la experiencia del Laboratorio de Innovación Transformativa (LIT) en la formación de investigadores como agentes de cambio en materia de sostenibilidad. La experiencia del LIT asume que una investigación es transdisciplinaria en la medida que aborda problemas relevantes para la sociedad, permite procesos de aprendizaje compartidos, integra conocimientos provenientes de disciplinas diversas y actores no académicos y, finalmente, tiene como propósito la generación de conocimiento sólido orientado a la solución de problemas científicos o sociales. En esta experiencia de formación es indispensable la articulación de los propósitos curriculares con los investigativos; se acude, por tanto, a la metodología de la investigación transdisiciplinaria (con sus tres fases para abordar el problema, integrar y reintegrar conocimiento), al enfoque de investigación contextualizado que ofrecen los laboratorios del mundo real (que se ocupa de la aplicación de conocimientos cocreados y aprendizajes cíclicos a partir de éstos), así como a los planteamientos de los estu-

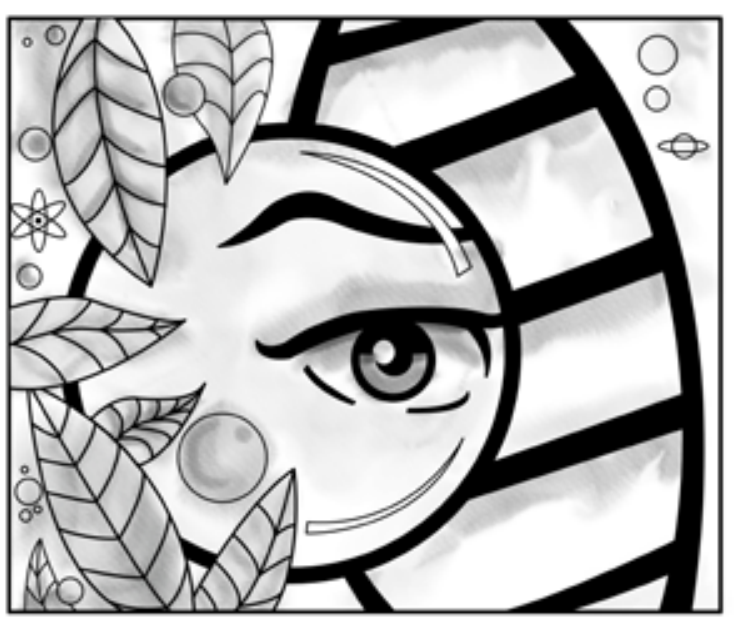

Transdisciplina y docencia universitaria. $\bullet 5$ Araceli Martínez Arroyo. DIDAC 78 (2021): 3-7 
dios del futuro (y sus herramientas para la toma de decisiones en futuros posibles de incertidumbre y sorpresa). En el pilotaje de esta experiencia en dos universidades alemanas se aprecian los logros de aprendizaje entre pares así como el beneficio de los momentos de implementación; al mismo tiempo, se enuncian los retos o necesidades futuras en relación con el seguimiento y evaluación del desarrollo personal, el aporte al debate científico y la promoción de prácticas sostenibles.

En "Comunidades de aprendizaje transdisciplinarias: cuidando lo común", Juliana Merçon describe el vínculo indisoluble entre la educación-investigación para el abordaje de lo transdisciplinar. En una revisión del concepto de la transdisciplina, Merçon adhiere y formula un planteamiento que asume a la educación-investigación transdisciplinaria como un "proceso que depende del cultivo de una comunidad de aprendizaje en la que interactúan diferentes sensibilidades ético-afectivas, capacidades epistémicas y visiones políticas" (Merçon, 2021). En el contexto de las instituciones de educación superior, dicho cultivo de comunidades de aprendizaje transdisciplinares emerge en la construcción de relaciones solidarias y de aprendizaje mutuo con agrupaciones de diferentes comunidades de práctica (empresas, gobierno, organizaciones de la sociedad civil, academia, entre otras) con las que se intercambian, integran y cogeneran conocimientos que permitan la comprensión compleja de la realidad así como las acciones para su transformación. La acción transformadora de esta relación plantea el cuidado de lo común como condición indispensable para el surgimiento de la diversidad y como práctica transgresora de una justicia epistémica desde la igualdad política.

En "De la teoría a la práctica transdisciplinar: lecciones aprendidas desde el quehacer académico universitario", José Manuel Maass Moreno explora los aprendizajes obtenidos en una trayectoria de más de tres décadas como docente e investigador que transita de una ciencia interdisciplinaria hacia aproximaciones más transdisciplinarias. Como el título del artículo indica, dichos acercamientos no pueden realizarse únicamente desde el ejercicio teórico, exi- gen acciones concretas que lo posibiliten. En la experiencia del autor ha sido fundamental desplazar el reduccionismo hacia una mirada sistémica, comprender la realidad socioecosistémica antes que al ecosistema y promover el pluralismo epistemológico antes que las explicaciones disciplinares (lo cual no es equivalente a sumar participantes de distintas disciplinas, sino a aumentar las aproximaciones para entender la realidad). Maass reconoce que la investigación y la enseñanza transdisciplinarias requieren ámbitos de trabajo en los que se practique la transversalidad, y en los que pongan en tensión aspectos tales como pertinencia versus rigor científico o ciencia aplicada versus básica. Las reflexiones compartidas por el autor, dan cuenta de que el camino hacia la transdisciplina no es sencillo, pero que es urgente andarlo ante nuestros modos de vida insustentables y nuestra necesidad de transformarlos. La creación de comunidades de aprendizaje transdiciplinares son, en este sentido, es una respuesta y una apuesta con la que merece la pena comprometerse.

La idea de hacer ciencia "con la sociedad", en vez de hacer ciencia "para la sociedad", es un giro indispensable para situarse y atender problemas sociales complejos y relevantes y, al mismo tiempo, para considerar en los procesos formativos del contexto universitario. Los autores de "Los caminos a la Inter y Transdisciplina de la Universidad Iberoamericana” asumen que la transdisciplina puede considerarse un enfoque-método-práctica que promueve la contribución activa y el consenso de personas más allá de la academia con saberes distintos a los provenientes de la ciencia occidental. Desde esta comprensión de lo transdisciplinar, el artículo ofrece un diagnóstico de los programas educativos en la Ibero y su visión inter y transdisciplinaria. Destacan no solamente ejemplos de planes de estudios diseñados desde este enfoque, seminarios o prácticas de trabajo docente, sino la creación de unidades académicas que impulsan dichos abordajes. En el tránsito de prácticas curriculares interdisciplinares y su aproximación a otras de carácter transdisciplinar, el artículo explora también algunos significados que docentes, coordinadores y directores otorgan sobre estos con- 
ceptos en sus programas académicos. Los autores advierten las necesidades de flexibilidad organizativas y administrativas que deben garantizarse institucionalmente y, finalmente, plantean cuatro propuestas clave para impulsar la inter y la transdisciplina al interior de la universidad: 1) participación y acción de todas las áreas que conforman la universidad; 2) diálogo a nivel conceptual; 3) flexibilizar procesos administrativos, y 4) contar con estructuras curriculares flexibles por parte de instancias educativas oficiales.

Finalmente, el artículo "El futuro del presente. El Seminario Permanente de Multi, Inter y Transdisciplina en la Universidad Iberoamericana: una respuesta a los nuevos retos de la educación superior", de Javier Cuesta y Berenice Pardo, describe una propuesta concreta que al interior de esta institución se construye con un grupo de académicos de diversas áreas y especialistas externos para detectar los múltiples caminos en que puede promoverse el tránsito a lo inter y transdisciplinario. Con base en una primera etapa de estudio orientada por especialistas externos sobre las implicaciones teóricas, epistemológicas y pedagógicas de estos conceptos, el Seminario ha conformado grupos de trabajo que, en torno a temas o problemas tales como la sustentabilidad, la desigualdad o la paz y gobernanza, han realizado documentos de estudio, diagnósticos de estos temas en programas académicos, o bien, propuestas concretas en diseño curricular. No obstante que este espacio recién se constituyó en 2019 y se ha visto afectado por las restricciones ocasionadas con la pandemia, sus propósitos y el ánimo de sus participantes lo han mantenido vivo y en vinculación activa con unidades académicas clave de la universidad. Sin duda, este Seminario se consolidará como un referente que permita que la Universidad Iberoamericana responda a su preocupación de relacionar los saberes impartidos con soluciones reales ante problemas sociales complejos.

\section{Agradecimientos}

Agradecemos la participación del Dr. José Alberto Lara Pulido en la coordinación de este número 78 de la revista $D I D A C$, así como al equipo del Centro Transdisciplinar Universitario para la Sustentabilidad (CENTRUS) y dictaminadores externos en la evaluación de los artículos.

\section{REFERENCIAS}

Benavides, M., et al. (2021). El curso en Educación Basada en Evidencia (EBE): reflexiones para la transdisciplina, la docencia y la investigación. DIDAC, 78(2021): 8-15.
Merçon, J. (2021). Comunidades de aprendizaje transdisciplinarias: cuidando lo común. DIDAC, 78(2021): 72-79.

\section{SEMBLANZA CURRICULAR}

Doctora en Educación y licenciada en Pedagogía por la Universidad Complutense de Madrid. Maestra y licenciada en Pedagogía por la Universidad Nacional Autónoma de México. Especialista en entornos virtuales de aprendizaje por la Organización de Estados

Iberoamericanos. Ha participado en actividades de edición de contenidos y recursos educativos, investigación pedagógica, formación docente y desarrollo curricular.

Actualmente es académica de tiempo completo adscrita al Programa de Desarrollo e Innovación Docente, responsable del proyecto de investigación "La dimensión social en el curriculum de la Universidad Iberoamericana” y editora de la revista DIDAC. 